\title{
Classification of Oral Submucous Fibrosis using SVM
}

\author{
S.Venkatakrishnan \\ Assistant Professor \\ Mechanical Engineering \\ Annamalai University
}

\author{
V.Ramalingam, Ph.D \\ Professor and Head \\ Computer Science and \\ Engineering \\ Annamalai University
}

\author{
S. Palanivel, Ph.D \\ Professor \\ Computer Science and \\ Engineering \\ Annamalai University
}

\begin{abstract}
Medical images form an essential source of information for various important tasks such as diagnosis of diseases, surgical planning, medical reference, research and training. Oral submucous fibrosis [OSMF] is a chronic debilitating disease of the oral cavity characterized by inflammation and progressive fibrosis of the submucosal tissues. Support Vector Machine $[\mathrm{SVM}]$ is a statistic machine learning technique that has been successfully applied in the pattern recognition and is based on the principle of structural risk minimization. In this paper a histogram based feature extraction technique has been proposed to classify normal images and OSMF affected images using SVM. An attempt is made to provide an enhanced knowledge about computer aided diagnosis of this potentially malignant disorder, to health care providers in order to help in differentiating the OSMF affected tissue from normal. Experiments showed significantly satisfactory results with an accuracy of $94 \%$.
\end{abstract}

\section{General Terms}

Pattern classification, computerized diagnosis, medical image processing

\section{Keywords}

Feature extraction, Histogram, Image classification, SVM.

\section{INTRODUCTION}

Oral submucous fibrosis is a chronic debilitating disease of the oral cavity characterized by inflammation and progressive fibrosis of the submucosal tissues (lamina propria and deeper connective tissues). Oral submucous fibrosis results in marked rigidity and an eventual inability to open the mouth. The buccal mucosa is the most commonly involved site, but any part of the oral cavity can be involved, even the pharynx.

The condition is well recognized for its malignant potential and is particularly associated with areca nut chewing, the main component of betel quid. Betel quid chewing is a habit practiced predominantly in Southeast Asia and India that dates back for thousands of years. It is similar to tobacco chewing in westernized societies. The mixture of this quid, or chew, is a combination of the areca nut (erroneously termed betel nut) and betel leaf (from the Piper betel, a pepper shrub), tobacco, slaked lime (calcium hydroxide), and catechu (extract of the Acacia catechu tree). Lime acts to keep the active ingredient in its freebase or alkaline form, enabling it to enter the bloodstream via sublingual absorption. Arecoline, an alkaloid found in the areca nut, promotes salivation, stains saliva red, and is a stimulant.
The pathogenesis of the disease is not well established. A number of factors trigger the disease process by causing a juxtaepithelial inflammatory reaction in the oral mucosa. Areca nut chewing, ingestion of chilies, genetic and immunologic processes, nutritional deficiencies, etc, are a few among them.

Symptoms of oral submucous fibrosis include:

- Progressive inability to open the mouth (trismus) due to oral fibrosis and scarring

- Oral pain and a burning sensation upon consumption of spicy foodstuffs

- Increased salivation

- Change of gustatory sensation

- Hearing loss due to stenosis of the eustachian tubes

- Dryness of the mouth

- Nasal tonality to the voice

- Dysphagia to solids (if the oesophagus is involved)

- Impaired mouth movements (eg, eating, whistling, blowing, sucking)

This paper is organized as follows: Section 2 gives a brief summary of related work done in the computerized diagnosis of oral diseases. Histogram Feature Extraction is described in Section 3. The principle of Support Vector Machine is described in Section 4. Experimental results are discussed in Section 5. Conclusion and future work are given in Section 6.

\section{RELATED WORK}

Application of Image Analysis methods in OSMF, other precancerous conditions and oral cancer for diagnosis, staging and classification aspects is being expertised in the recent years. R.R Paul et al [in 2005] described a novel neural network for OSF staging [15]. The wavelet coefficients of transmission electron microscopy images of collagen fibres from normal oral submucosa and OSF tissues were used to choose the feature vector which in turn, was used to train the artificial neural network. Similarly, Mukherjee.A, ,R.R.Paul et al. [in 2006] used a three-layered feed-forward neural network and the feature vector, formed by calculating the wavelet coefficients[13]. Four wavelet decomposition functions, namely GABOR, HAAR, DB2 and DB4 were used. Textural Analysis of Spinous Layer for Grading Oral Submucous Fibrosis was performed by Rusha Patra and Chandan Chakraborty [in 2012]. The epithelium was segmented using anisotropic diffusion and Otsu's thresholding [14]. Wavelet based multi-resolution technique was applied to extract 12 textural features from spinous layer. Support vector machine (SVM) and Bayesian classifiers were trained.

M.MuthuRamakrishnan et al. [in 2009] classified the subepithelial connective tissue cells of OSMF [7]. Segmentation and classification was done with Support Vector Machine 
[SVM] based classifier. An automated classification of the OSMF cells with their geometric features was presented, giving an understanding about the level of deviation from the normal structure. M.E.Tathagata Ray et al. [in 2008] performed segmentation of OSMF images into its constituent layers using Hybrid Segmentation Algorithm [HSA] and was proved better over Region Growing Algorithm [RGA][6]. Later a texture based epithelial layer segmentation algorithm was applied by Muthu Rama Krishnan et al [in 2011]. Watershed segmentation was used to segment layers with texture and color gradients of both normal and OSMF mucosae[9]. OSMF with and without dysplasia was differentiated using In an attempt to develop an automated diagnostic methodology for OSMF. Also collagen fibres were used for feature extraction and classifiers used were Bayesian classification and SVM by MuthuRamaKrishnan [in 2012][8]. Here Gaussian transformation improved the results in classifying normal and OSMF cases. MuthuRama Krishnan et al [in 2013] did feature extraction using Brownian movement curve [BMC] to classify OSMF vs normal[11]. Along with a textural classifier designed using BPNN, this yielded an appreciable accuracy of $96.43 \%$ in diagnosis.

\section{FEATURE EXTRACTION 3.1 Feature Selection}

The feature is defined as a function of one or more measurements, each of which specifies some quantifiable property of an object, and is computed such that it quantifies some significant characteristics of the object. Feature selection helps to reduce the feature space which improves the prediction accuracy and minimizes the computation time. Quantitative evaluation of histopathological features is not only vital for precise characterization of any precancerous condition but also crucial in developing automated computer aided diagnostic system. Sub-epithelial hyalinization and fibrosis are characteristic histological features of OSMF. In this study, color histogram features were extracted from both normal and OSMF microscopic images. Classification was done using SVM.

\subsection{To construct a histogram}

In statistics, a histogram is a graphical representation of the distribution of data. It is an estimate of the probability distribution of a continuous variable. A histogram is a representation of tabulated frequencies, shown as adjacent rectangles, erected over discrete intervals (bins), with an area equal to the frequency of the observations in the interval. The height of a rectangle is also equal to the frequency density of the interval, i.e., the frequency divided by the width of the interval. The total area of the histogram is equal to the number of data. A histogram may also be normalized displaying relative frequencies. It then shows the proportion of cases that fall into each of several categories, with the total area equaling 1 . The categories are usually specified as consecutive, non-overlapping intervals of a variable. The categories (intervals) must be adjacent, and often are chosen to be of the same size. The rectangles of a histogram are drawn so that they touch each other to indicate that the original variable is continuous.

Histograms are used to estimate the probability density function of the underlying variable. The total area of a histogram used for probability density is always normalized to 1. If the lengths of the intervals on the $\mathrm{x}$-axis are all 1 , then a histogram is identical to a relative frequency plot.
Color histogram is used to compare images in many applications. In this study, RGB color space is quantized into 64-dimensional feature vectors which are used as features. The image histogram is a simple bar graph of pixel intensities. The pixels are plotted along the $\mathrm{x}$-axis and the number of occurrences for each intensity represents the y-axis:

$$
p\left(n_{k}\right)={ }^{n_{k}} /_{n_{v}} \quad 0 \leq k \leq L-1
$$

where,

$$
\begin{array}{ll}
r_{k}- & k^{\text {th }} \text { gray level } \\
n_{k} & \text { no. of pixels in the image with that gray } \\
& \text { level. } \\
\mathrm{L}- & \text { no. of levels } \\
n & - \text { total no. of pixels in the image } \\
\mathrm{p}\left(r_{k}\right) & \text { gives the probability of occurrence of gray } \\
& \text { level } \mathrm{r}_{\mathrm{k}}
\end{array}
$$

Divide the range between the highest and lowest values in a distribution into several bins of equal size. Toss each value in the appropriate bin of equal size. The height of a rectangle in a frequency histogram represents the number of values in the corresponding bin.

\section{CLASSIFIERS}

After the features are extracted, a suitable classifier must be chosen. A number of classifiers are used and each classifier is found suitable to classify a particular kind of feature vectors depending upon their characteristics. The classifier used commonly is Support Vector Machine. The SVM classifier is used to compare the feature vector of the prototype with image feature vectors stored in the database. It is obtained by finding the distance between the prototype image and the database.

\subsection{Support Vector Machines (SVM)}

SVM is a statistic machine learning technique that has been successfully applied in the pattern recognition area and, is based on the principle of structural risk minimization . SVM constructs a linear model to estimate the decision function using non-linear class boundaries based on support vectors. SVM learns an optimal separating hyperplane from a given set of positive and negative examples. It minimizes the structural risk, that is, the probability of misclassifying yet-tobe-seen patterns for a fixed but unknown probability distribution of the data. This is in contrast to traditional pattern recognition techniques of minimizing the empirical risk, which optimizes the performance on the training data.

For linearly separable data, SVM finds a separating hyperplane which separates the data with the largest margin, without error and into the maximum distance between the hyperplane and the closest training points. The training points that are closest to the optimal separating hyperplane are called support vectors. For linearly inseparable data, it maps the data in the input space into a high dimension space $\mathrm{x} \in \mathrm{R} \rightarrow \Phi(\mathrm{x})$ $\in \mathrm{R} \mathrm{H}$ with kernel function $\Phi(\mathrm{x})$, to find the separating hyperplane.

SVMs are evaluated as popular tools for learning from the given data. The reason is that, SVMs are more effective than the traditional pattern recognition approaches based on the combination of a feature selection procedure and a conventional classifier. SVM was originally developed for two class classification problems. The $\mathrm{N}$ class classification problem can be solved using N SVMs. Each SVM separates a 
single class from all the remaining classes (one-vs-rest approach).

Fig. 1 shows the architecture of the SVM. SVM maps the input patterns into a higher dimensional feature space through some nonlinear mapping chosen a priori. A linear decision surface is then constructed in this high dimensional feature space. Thus, SVM is a linear classifier in the parameter space, but it becomes a non-linear classifier as a result of the nonlinear mapping of the space of the input patterns into the high dimensional feature

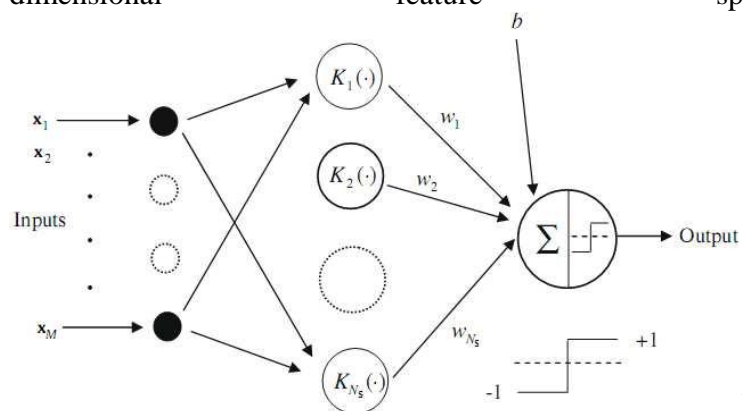

Fig.

1: Architecture of the SVM (Ns is the number of support vectors).

The kernel function may be any of the symmetric functions that satisfy the Mercer's conditions (Courant and Hilbert,1953). There are several SVM kernel functions as given in Table 1.

Table 1: Types of SVM inner product kernels.

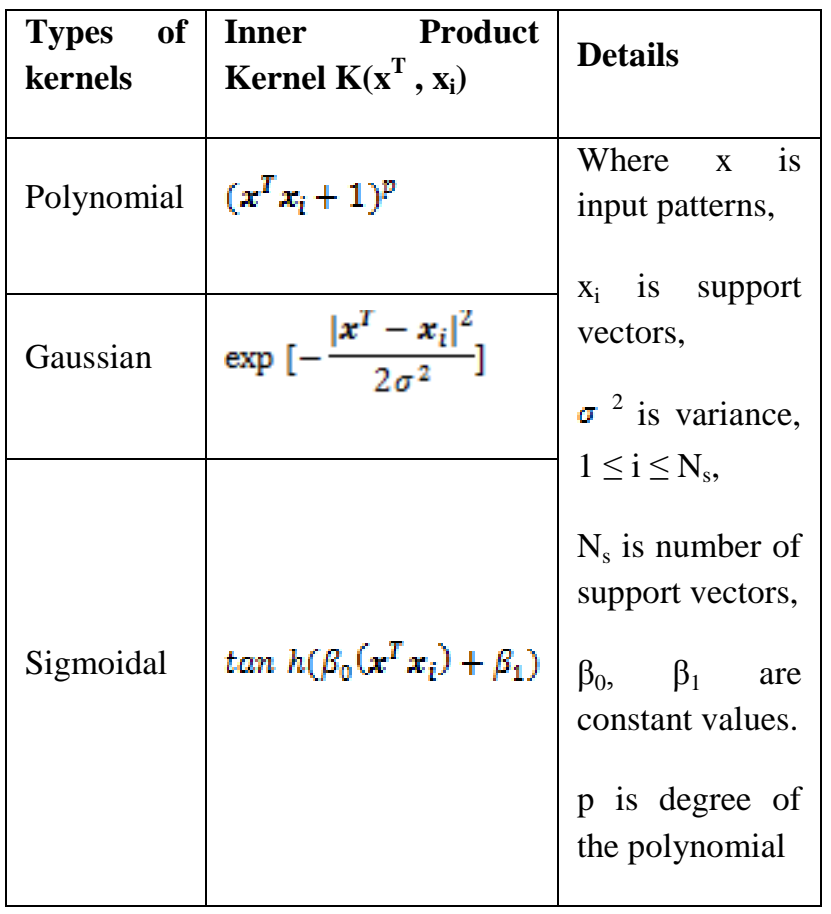

\section{RESULTS AND DISCUSSION}

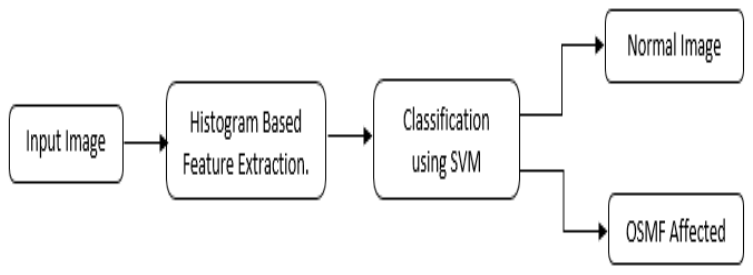

Fig. 2 Block Diagram of the Classification System.

Fig 2 shows the overall architecture of the proposed classification system.

Normal and OSMF affected tissue images were collected from patients of Raja Muthiah Dental College and Hospital (RMDC \& H). Microscopic images of 30 normal oral mucosal tissue samples and 30 OSMF affected images were used for training the (SVM) classifier.

Histogram features were extracted from both normal and OSMF affected images. Repeated experiments were carried out for 16, 32 and 64 bins which resulted in 16, 32 and 64 dimensional feature vectors. These feature vectors where used to train the support vector machine. For testing the histogram features were extracted from the test image and given as input to SVM which classifies the input image into one of the predefined categories (normal or OSMF affected image). 4fold cross-validation was applied to a data set consisting of 120 feature vectors, resulting in 4 equal sized sub-samples. Out of the 4 sub-samples, a single sub-sample is retained as the validation data for testing the model and the remaining 3 sub-samples were used as training data. Cross validation process was repeated 4 times and performance of the system was analyzed.

Fig. 3 shows the performance of OSMF classification for different bins.

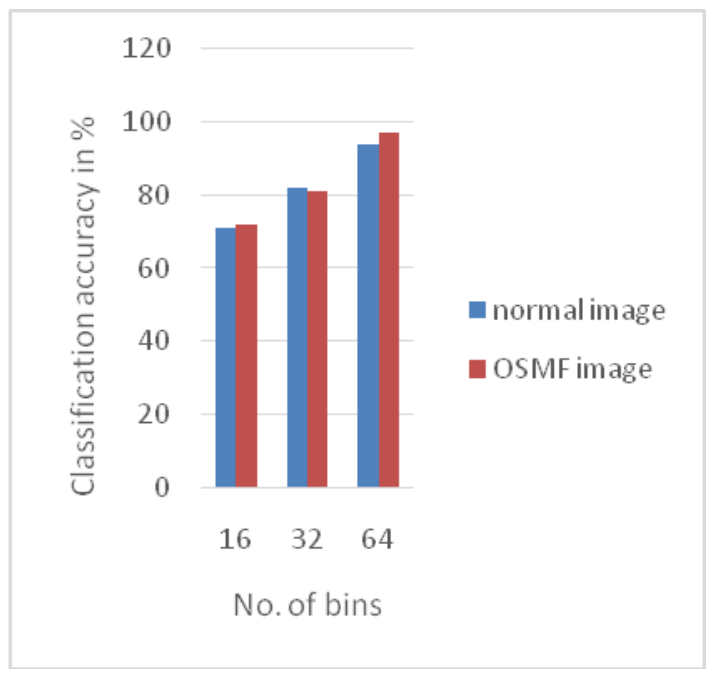

Fig. 3 Performance of SVM for OSMF classification 
The above figure shows that maximum performance is achieved for 64 bins. Repeated experiments were carried out for 16,32 and 64 bins.

Table 2: shows the performance of OSMF classification for different kernel function using SVM.

Table 2: Performance comparison of OSMF classification in terms of different kernel functions using SVM

\begin{tabular}{|l|c|c|c|}
\hline \multirow{2}{*}{ Type of kernel } & \multicolumn{3}{|c|}{ Number of bins } \\
\cline { 2 - 4 } & 16 & 32 & 64 \\
(in \%) & (in \%) & (in \%) \\
\hline Polynomial & 79 & 82 & 88 \\
\hline Gaussian & 89 & 91 & 94 \\
\hline Sigmoidal & 71 & 77 & 80 \\
\hline
\end{tabular}

\section{CONCLUSION}

In this paper, a system for classifying OSMF affected images from normal images was proposed. Color histogram features where extracted from both normal and OSMF affected images. The features where trained and tested using SVM for different bins. The system showed an accuracy of $97.0 \%$ for 64 bins. In future other pattern recognition algorithms can be analyzed and the performance can be studied for computerized diagnosis of OSMF.

\section{ACKNOWLEDGEMENT}

We would like to thank with overwhelmed gratitude, the enormous support and guidance rendered to us by Dr. P.Dhanalakshmi, Associate Professor, Dept. of Computer Science and Engineering, Annamalai University.

\section{REFERENCES}

[1] Van der Waal.I,Potentially malignant disorders of the oral and oropharyngeal mucosa;present concepts of management,Oral Oncology,46(6):423-5,June 2010.

[2]Kunio Doi. Computer-aided diagnosis in medical imaging:historical review, current status and future potential. Journal-Computerised medical imaging and Graphics, 31:198-211, 2007.

[3]M.M.R.Krishnan, .R. Acharya,C. Chakraborty, A. K. Ray, Automated Diagnosis of Oral Cancer Using Higher Order Spectra Features and Local Binary Pattern: A Comparative Study TCRT , 10(5):391-504,October 2011.

[4]Shah.P. Sch. of Med. Sci. \& Technol., IIT,- Kharagpur, Kharagpur, India ; Krishnan, M.M.R. Chakraborty, C, Ray, A.K., Morphometric Pattern Analysis of Basal Cell
Nuclei for Oral Cancer Screening, Bioinformatics and Biomedical Engineering [iCBBE] 2010 National conference, 1-4, June 2010.

[5] Ray AA Krishnan.MM.Shah.P, Choudhary AA.Chakraborty.C.Paul RR. Textural characterization ofhistopathological images for oral sub-mucous fibrosisdetection. Tissue cell, 43(5):318-30, October 2011.

[6] Anirban Mukherjee Jyotirmoy Chatterjee Ranjan.R.Paul Pranab.K.Dutta, M.E.Tathagata Ray, D.Shivashanker Reddy. Detection of constituent layers of histological oral sub-mucous fibrosis images using the hybrid segmentation algorithm. Oral Oncology, 44(12):11671171,December 2008.

[7]M.Muthuramakrishnan,Pal,Suneel.K.Bomminayuni,Chand an Chakraborthy, Automated classification of cells in sub-epithelial connective tissue of oral sub-mucous fibrosis-An SVM based approach.Computers in Biology and medicine,39(12):1096-1104. December 2009.

[8]Chandan .K.Ray M.MuthuRamaKrishnan,PratikShah. Statistical analysis of textural features for improved classification of oral histopathological images. Journal of Medical Systems, 36(2):865-881, April 2012.

[9]

$$
\text { Chandan Chakraborty Ajoy.K.Ray }
$$

Ranjan.R.PaulM.MuthuRamaKrishnan, Anirudh Choudhry. Texturebased segmentation of epithelial layer from oral histological images. Micron, 42(6):632-641, August 2011.

[10] U.Rajendra Acharya, Mousumi Pal, Ranjan Rashmi Paul, LimChoo Min Ajay kumar Ray Jyotirmoy Chatterjee Chandan Chakraborty M.Muthuramakrishnan, Vikram Venkatraghavan. Automated oral cancer identification using histopathological images: A hybrid feature extractionparadigm. 43(2-3):352-364, February 2012.

[11] Pratik Shah M.Tech. Chandan Chakraborty- MuthuRama Krishnan Mookiah,s Ajoy K. Ray.Brownian motion curvebased textural classification and its application in cancer diagnosis. Analytical and Quantitative Cytology and Histology, , May 2013.

[12] MuthuramaKrishnan.M.Shah. P, Structural markers for normal oral mucosa and oral sub-mucous fibrosis. Micron, 41(4):312-20, June 2010.

[13] Anirban Mukherjee,Ranjan Rashmi Paul,Keya Chaudhuri,Jyotirmoy Chatterjee,Mousumi Paul,Provas Banerjeee,kanchan Mukherjee,Swapna, Performance analysis of different wavelet feature vectors in quantification of oral precancerous condition, Oral Oncology,42(9):914-28, October 2006.

[14]Jsyotirmoy Chatterjee Rusha Patra, Chandan Chakraborty.Textural analysis of spinous layer for grading oral submucousfibrosis ,. International Journal of Computer Applications, 47:975-8887,June 2012.

[15] R R Paul, A Mukherjee, P K Dutta, S. Banerjee, M Pal, J Chatterjee, K. Chaudhuri, K. Mukkerjee,A novel wavelet neural network based pathological stage detection technique for an oral precancerous condition, J.Clin Pathol,58(2):932-938,February 2005. 